\title{
Laser-Based
}

\section{Characterization of \\ Nuclear Fuel \\ Plates}

\section{0th Annual Review of Progress in Quantitative Nondestructive Evaluation Conference}

\author{
James A. Smith \\ Dave L. Cottle \\ Barry H. Rabin
}

The INL is a

U.S. Department of Energy

National Laboratory

operated by

Battelle Energy Alliance

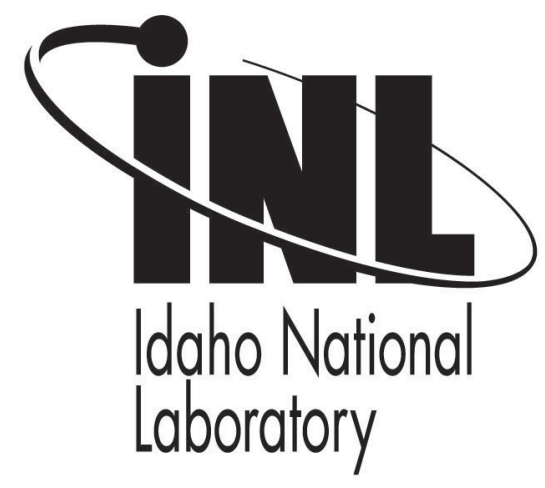

\section{July 2013}

This is a preprint of a paper intended for publication in a journal or proceedings. Since changes may be made before publication, this preprint should not be cited or reproduced without permission of the author. This document was prepared as an account of work sponsored by an agency of the United States Government. Neither the United States Government nor any agency thereof, or any of their employees, makes any warranty, expressed or implied, or assumes any legal liability or responsibility for any third party's use, or the results of such use, of any information, apparatus, product or process disclosed in this report, or represents that its use by such third party would not infringe privately owned rights. The views expressed in this paper are not necessarily those of the United States Government or the sponsoring agency. 


\title{
Laser-Based Characterization of Nuclear Fuel Plates
}

\author{
James A. Smith, Dave L. Cottle, and Barry H. Rabin \\ Idaho National Laboratory, Fuel Performance and Design, P.O. Box 1625, Idaho Falls Idaho, 83415-6188
}

\begin{abstract}
Ensuring the integrity of fuel-clad and clad-clad bonding in nuclear fuels is important for safe reactor operation and assessment of fuel performance, yet the measurement of bond strengths in actual fuels has proved challenging. The laser shockwave technique (LST) originally developed to characterize structural adhesion in composites is being employed to characterize interface strength in a new type of plate fuel being developed at Idaho National Laboratory (INL). LST is a non-contact method that uses lasers for the generation and detection of large-amplitude acoustic waves and is well suited for application to both fresh and irradiated nuclear-fuel plates. This paper will report on initial characterization results obtained from fresh fuel plates manufactured by different processes, including hot isostatic pressing, friction stir welding, and hot rolling.
\end{abstract}

Keywords: Laser Shockwave, Nuclear Fuel, Aluminum Cladding, Uranium Alloys, Characterization, Clad-clad Bond Strength, Fuel-clad Bond Strength, Fabry-Perot Detection.

PACS: *43.60.Vx, 28.41.Rc, 28.41.Bm, 46.40.Cd, 65.40.De

\section{INTRODUCTION}

The US National Nuclear Security Agency oversees the Global Threat Reduction Initiative (GTRI), which is tasked with minimizing the use of highly enriched uranium (HEU) worldwide. A key component of that effort is the conversion of research reactors from HEU to low-enriched uranium (LEU) fuels. A novel fuel is being designed to replace HEU. The new LEU fuel is based on a monolithic fuel meat made from a uranium-molybdenum (U-Mo) alloy foil encapsulated in Al-6061 cladding, with thin $\mathrm{Zr}$ diffusion barrier interlayers between the U-Mo and cladding. In order to support the fuel qualification process, new techniques are needed to characterize the clad-clad and fuel-clad interface strengths.

As shown schematically in Fig. 1a, the monolithic fuel is based on a rolled U-Mo alloy foil (typically 0.2 to 0.4 $\mathrm{mm}$ thick) that is covered with a thin (typically $25 \mu \mathrm{m}$ thick) Zr diffusion barrier layer and then clad in Al-6061 by a hot isostatic pressing (HIP) process. This monolithic fuel has shown promising results in irradiation testing.

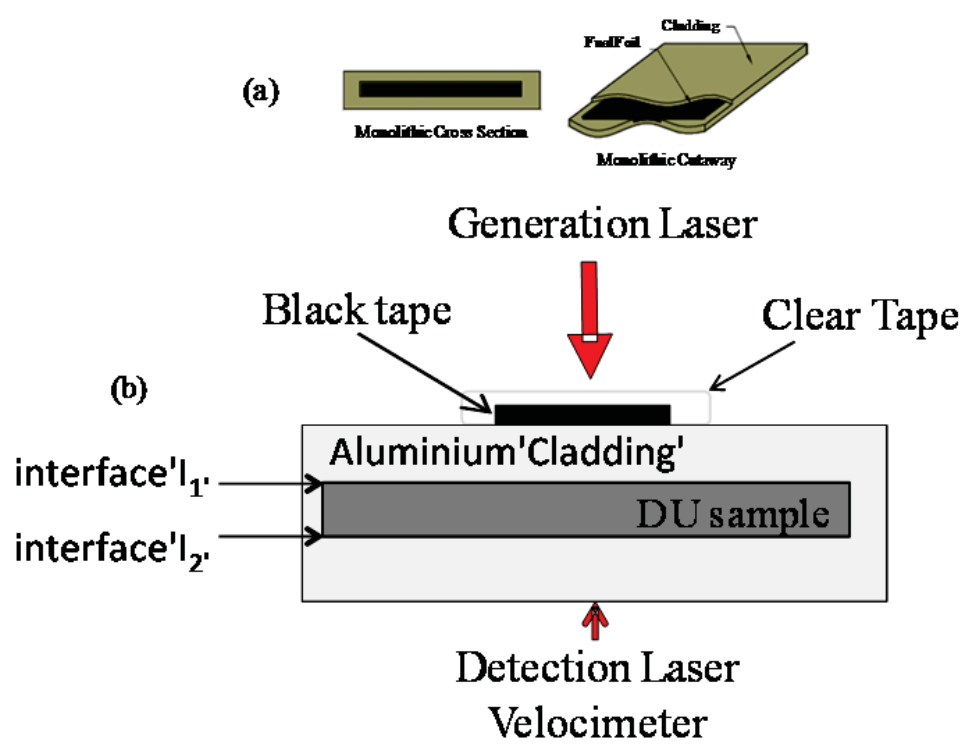

FIGURE 1. Schematic showing the specimen (a) and the geometries for laser shock generation, plasma constraining mechanism and back surface velocity detection (b). 
Monolithic fuel is fundamentally different from historical dispersion fuels used in most research reactors. The fuel-clad interface has a $\mathrm{Zr}$ diffusion barrier that has a complex microstructure which evolves over time. The mismatch in properties across interfaces results in localized stresses during fabrication and irradiation. Similar to dispersion fuels, the clad-clad interface in monolithic fuel provides the primary barrier for fission-product retention. However, while the new HIP manufacturing process produces fuel plates that pass traditional ultrasonic testing (UT) and bend-test inspections, they do not meet the historical criteria for grain growth across the interface that was used to qualify the previous roll-bonding manufacturing process. Therefore, a critical need exists to establish new evaluation methods and criteria supporting fuel qualification, manufacturing process development, and manufacturing process qualification.

Monolithic LEU fuel has recently transitioned from the research phase to development and qualification stages. One of the significant fuel-performance requirements that must be addressed involves ensuring that the clad-clad and fuel-clad interface strengths within the fuel plates are sufficiently high to maintain integrity throughout irradiation. Measurement of bond strengths before and after irradiation will provide valuable information in this regard. Furthermore, quality assurance requirements in the manufacturing process demand that suitable inspection and testing techniques are available to evaluate interfacial bond strengths in as-fabricated fresh fuel plates. This report is concerned primarily with bond-strength characterization. Past efforts to characterize bond strengths in monolithic fuel plates using conventional mechanical testing methods have been unsuccessful. The LST technique is being developed to provide needed capabilities.

LST has been used previously for the measurement of bond strength in thin coatings on a substrate [1-2]. For thin-coating measurements, a pulsed laser is used to generate a large-amplitude compression pulse which travels through the specimen as a shock wave. After reaching the back side of the specimen, the shock wave is reflected from the free surface as a tensile pulse that can debond the coating. Current research addresses the characterization of adhesive bonding in thicker structures composed of carbon-epoxy composite [3]. An advantage offered by LST is to provide a spatially localized measurement without contact. This allows the LST operator to remotely characterize a fuel plate in safety. Another benefit is that there is no propagation of the induced debond outside of the test area; thus, the specimen can be measured in many locations. The cladding remains intact and the fuel remains contained. Thus, the operator and equipment are protected against radioactive contamination that can occur with destructive testing. LST is not dependant on specimen geometry and surface roughness. LST does have unique features in characterizing bonding as compared to typical destructive techniques (such as pull testing, lap shear, double cantilever beam or mixed-mode flexure techniques). LST is a high-strain-rate technique, and the stresses developed are different from quasi-static deformations. The critical stress to create a debond (i.e. the bond strength) measured by LST may be on the order of 10 times greater than the quasi-static value. Another difference is that the bond strength measured by LST arises from a transient localized tensile stress acting normal to the interface while other destructive tests generally involve more complex stress states.

In the research discussed, LST has been adapted to the characterization of interfaces in nuclear fuel plates fabricated by HIP, roll bonding, and friction stir welding processes. Both clad-clad and fuel-clad interfaces have been characterized. The details of LST equipment and test methodology for bond strength characterization will be discussed. Results show that LST is able to distinguish weak and strong bonds resulting from the different manufacturing processes.

\section{PRINCIPLES AND EXPERIMENTAL APPROACH}

A Q-Switched neodymium doped: yttrium aluminum garnet (Nd:YAG) laser, which generates optical pulses of about $10 \mathrm{~ns}$ with a maximum energy of $3.5 \mathrm{~J}$ at $1064 \mathrm{~nm}$ wavelength, is used to induce shock waves to characterize the fuel plates. The laser beam is focused to a beam diameter near $5 \mathrm{~mm}$. To avoid surface damage and to increase the efficiency of optical-to-mechanical transduction [2,3], the surface of the material is covered with an absorbing tape and then covered with a transparent constraining medium (such as tape) as shown in Fig. 1b. The shock waves generated under the confinement layer produce large-amplitude molecular displacement rather than laser ablating the sample surface. Previous work [2-5] used a liquid-constraining medium. The liquid-constraining medium works well, but the liquid overspray can contaminate equipment and optics. To keep the water and plasma particulates contained, high-temperature transparent tape is used. The containment of the plasma particulates by the tape is more conducive to a manufacturing process.

The shock-wave source size (roughly the laser spot size) needs to be about two times the sample thickness (about $1.5 \mathrm{~mm}$ ) to approximate one-dimensional (1-D) wave propagation. Under the 1-D approximation, shear stresses are neglected, and the shock wave is exclusively compressive. The generated shock wave is then reflected by the back 
surface of the plate and converted to a tensile wave. Tensile stresses are responsible for debonding at the interface. The resulting stresses developed in the material may be the result of several shock waves besides the wave reflected from the back surface. Previous papers $[4,5]$ discuss a strategy to account for multiple reflections from interfaces within the specimen. This paper will use the simplifying assumption that the shock-wave energy reaching the backside is fully reflected and is the sole cause of the tensile stress at specimen interfaces. This simplifies the discussion and the resulting approximate stresses are adequate to illustrate the laser-shock technique. Bond strength is determined by increasing the laser pulse energy step-by-step. The ultimate stress value is imposed at the interface when debonding is imminent. To limit cumulative effects of plastic deformation in the material, a single shock is applied at each shock location. However, since shock waves are reflected from each surface or interface, the reflected shock wave propagates in material that has been subject to prior plastic deformation. Thus, plastic effects can occur during a single shock event. The stress is indirectly calculated from the measured back-wall surface velocity, u, (see Fig. 1b) recorded by an optical velocimeter based on a solid Fabry-Perot etalon. The velocimeter laser is a long pulse $(>120 \mu \mathrm{s}) \mathrm{Nd}$ :YAG, operated at $1064 \mathrm{~nm}$ wavelength. Details of the etalon interferometer can be found in reference [6].

The simplified relation between the back-wall surface velocity and stress is given by Eq. 1 .

Where,

$$
\sigma(t)=\rho c u(t)
$$

$c \quad=$ Speed of sound in $\mathrm{Al}(6.4 \mathrm{~mm} / \mu \mathrm{s} ; 6,400 \mathrm{~m} / \mathrm{s} ;$ measured $)$

$\rho=$ Al 6061 density $\left(2.70 \mathrm{~g} / \mathrm{cm}^{3} ; 2,700 \mathrm{~kg} / \mathrm{m}^{3}\right)$

$u(t)=$ Measured surface velocity $(\mathrm{m} / \mathrm{s})$

The first order estimate of bond strength is obtained by measuring the back-surface velocity just below the debond threshold, assuming an elastic 1-D wave propagation in which $100 \%$ of the energy is converted to a tensile wave and returns to the interface (i.e., neglecting attenuation) without multiple reflections or shock-wave overlapping. Despite the elastic propagation assumption, part of the plastic effect is compensated by using the velocity signal recorded at the back surface. Plastic deformation absorbs energy from the shock wave and reduces the surface velocity. Because of the intractable nature of a laser-generated shock wave from confined plasma, optical energy of the source laser is not an accurate predictor of shock-wave stress. The back-surface velocity is a more accurate representation of the stress imparted to the specimen. The stress estimate based on the back-surface velocity is reasonable as a first approximation. More detailed stress estimates may be obtained either by accounting for internal reflections from the different layers within the fuel plate in equation (1) or by numerical simulation of the shock wave propagation. These approaches are the subjects of future investigation.

Laser-ultrasonic C-scan measurements made prior to and after the laser shock are used to confirm the presence of debonds, as well as to determine the size and locate the depth of the debond created. These ultrasonic measurements are obtained from another laser-based experimental technique, where generation and detection laser beams are on the same surface, superimposed with diameter sizes of about $1 \mathrm{~mm}$ and $0.5 \mathrm{~mm}$, respectively. The step size of the scan is approximately $0.5 \mathrm{~mm}$ in the $\mathrm{x}$ and $\mathrm{y}$ directions on the sample surface. The generation laser was a Nd:YAG, operated at $532 \mathrm{~nm}$ wavelength with a full width at half maximum (FWHM) of $10 \mathrm{~ns}$. The detection uses a long pulse $(>120 \mu \mathrm{s}) \mathrm{Nd}$ :YAG laser, operated at $1064 \mathrm{~nm}$ wavelength and a photorefractive interferometer. The laserultrasonic inspection is performed without the tape or confinement and is similar to a conventional ultrasonic Cscan.

\section{EXPERIMENTS AND RESULTS}

Laser shock testing has been performed on fuel plates fabricated by three different clad bonding techniques: HIP, roll-bonded and friction stir welding. The first HIP example discussed below is used to introduce the laser shock technique and the data processing/display techniques used to characterize the shock locations in the specimen. Data obtained from the three fuel plate types are then presented for comparative purposes.

LST testing of a HIP fuel plate with a stainless steel foil is shown in Fig.2a. The fuel plate is an early prototype stainless steel (SS) surrogate fuel plate. This particular plate was made with Neolube parting agent (as an alternative to the Grafoil parting agent that is currently used) to keep the plate from bonding to the spacers in the HIP can. The Neolube approach was later abandoned because Neolube was thought to have contributed to weakening of interfacial bonds. Circular features on the fuel plate (Fig, 2a) show locations of laser shock testing. Dark black locations indicate areas of debond. 
Figure $2 \mathrm{~b}$ shows ways in which the data are displayed to characterize the effects of the LST. The A-scans are the raw laser ultrasonic signals taken at a single location. The B-scan images are a waterfall plot of A-scans taken along a line. C-scan images are the maximum value from each A-scan generated while the specimen is being scanned and imaged. The A-scans emphasize individual reflections and pulse shapes. B-scans emphasize relative timing and magnitude of the ultrasonic reflections. C-scans emphasize geometry and relative magnitude of return signals.

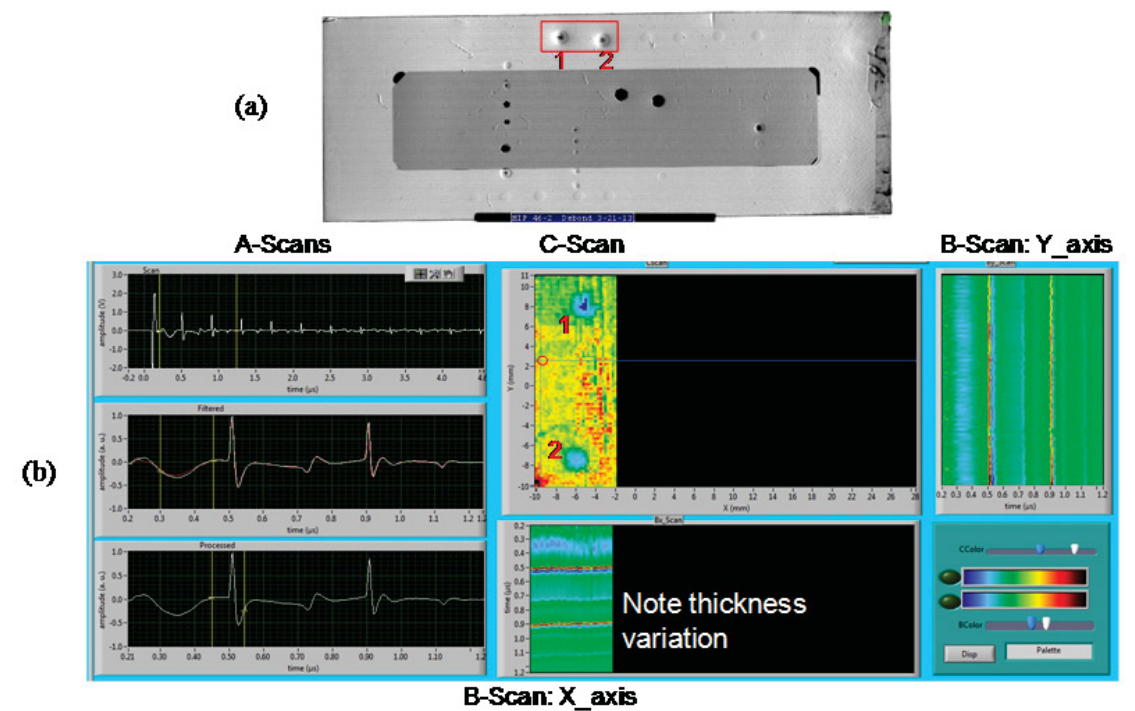

FIGURE 2. The laser shock locations being characterized are shown in the conventional ultrasonic C-scan (a). The laser ultrasonic processing necessary to characterize the laser shock locations is displayed (b). Signals at cursor location (circle) show good bonding and thinning of plate near edge of cladding (B-scan: X_axis image).

The cross-hair cursor (highlighted with a circle on the left) shown in the C-scan image of Figure $2 \mathrm{~b}$ was positioned on a well bonded region in a clad-only area. The B-scan images show contiguous lines formed from multiple back-wall reflections. The slope in the lines indicates that the plate thickness is decreasing as the edge of the plate is approached. The A-scans show the repetitive pattern of multiple back-wall reflections. C-scans identify the laser-shock locations and define the affected areas. The images in Fig. $2 \mathrm{~b}$ provide the baselines for comparison.

Figure 3 shows the resulting signals when there is a debond in the clad-clad interface, as indicated by the cursor position. The regularity and order of the reflections in the A-scans are missing. The dark signal in the middle A-scan is a baseline A-scan taken in a well bonded region. The B-scans also show additional interface reflections from debonds that are distinctly different from the well bonded areas.

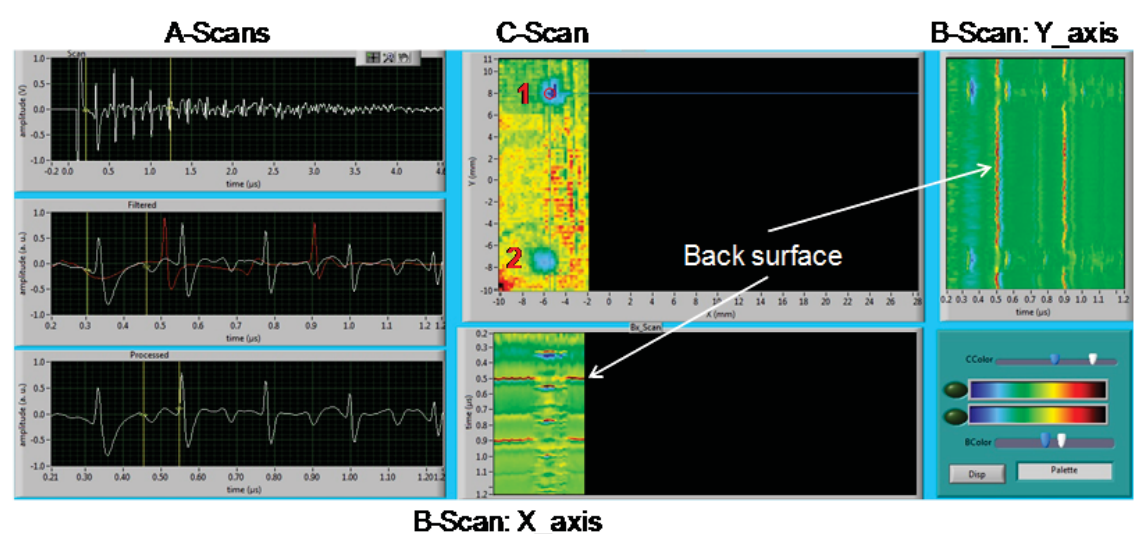

FIGURE 3. The three types of ultrasonic scans that are displayed show damage at laser shock location 1 . The damaged interface adds a reflected return signal closer to the surface and prevents the back surface from receiving ultrasonic energy, hence the break in the back-surface echo line. 
Figure 4 shows the resulting signals when there is a well bonded fuel-clad interface (b) and a debonded fuel-clad interface (c). In all cases, the fuel-clad interface complicated the resulting images. The debonded images show even more disorder. The A-scans show a signal inversion from the fuel-clad debond in the debonded images (Fig. 4c). The first and third reflections show an amplitude inversion in the return signals when compared to the dark baseline signal.

A roll bonded dispersion fuel plate specimen has been laser-shock tested at three locations using the maximum attainable laser energy, and the results are shown in Figure 5. A large blister can be seen in the traditional C-scan image. The presence of the blister indicates that the clad-clad bonding may be weak. LST suggests that the bonding improves in the section of the plate away from the blister as the laser-shock location (A) did not debond.

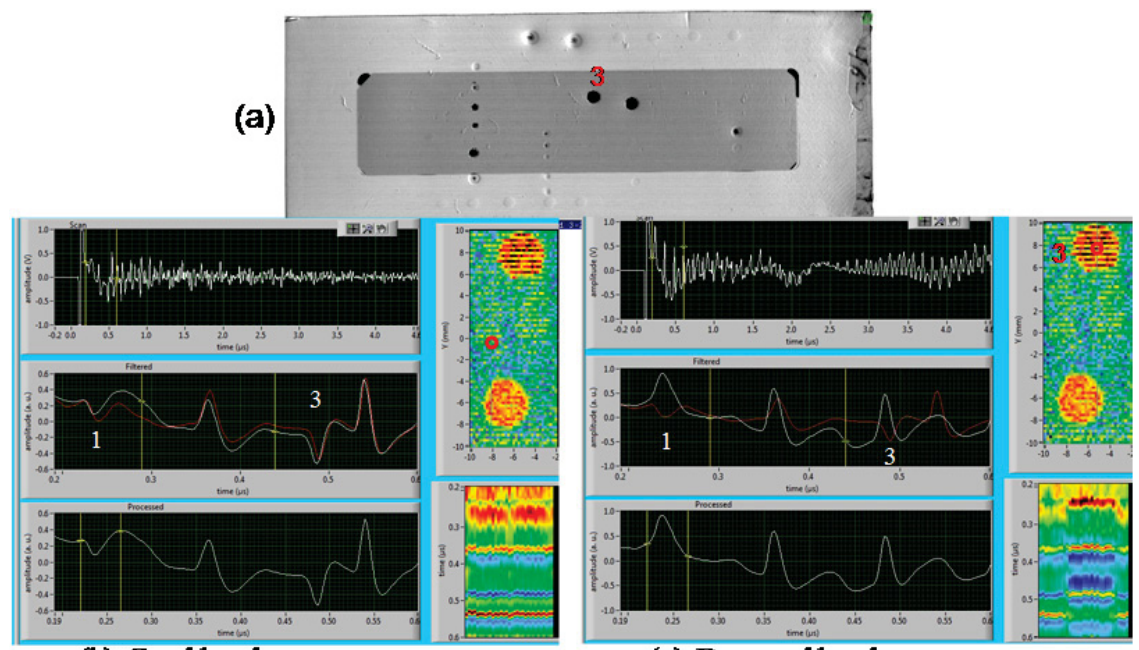

(b) Good bond

(c) Damaged bond

FIGURE 4. Laser C-scan data which shows the signal over a well bonded region (b) and over a damaged location from laser shock (c). In the expanded A-scan graph (middle image) for the damage location (c), the first and third arrivals are inverted. The darker signal is the baseline from a well bonded region. Signal inversion is clear evidence of debonding and reflection from a free surface.

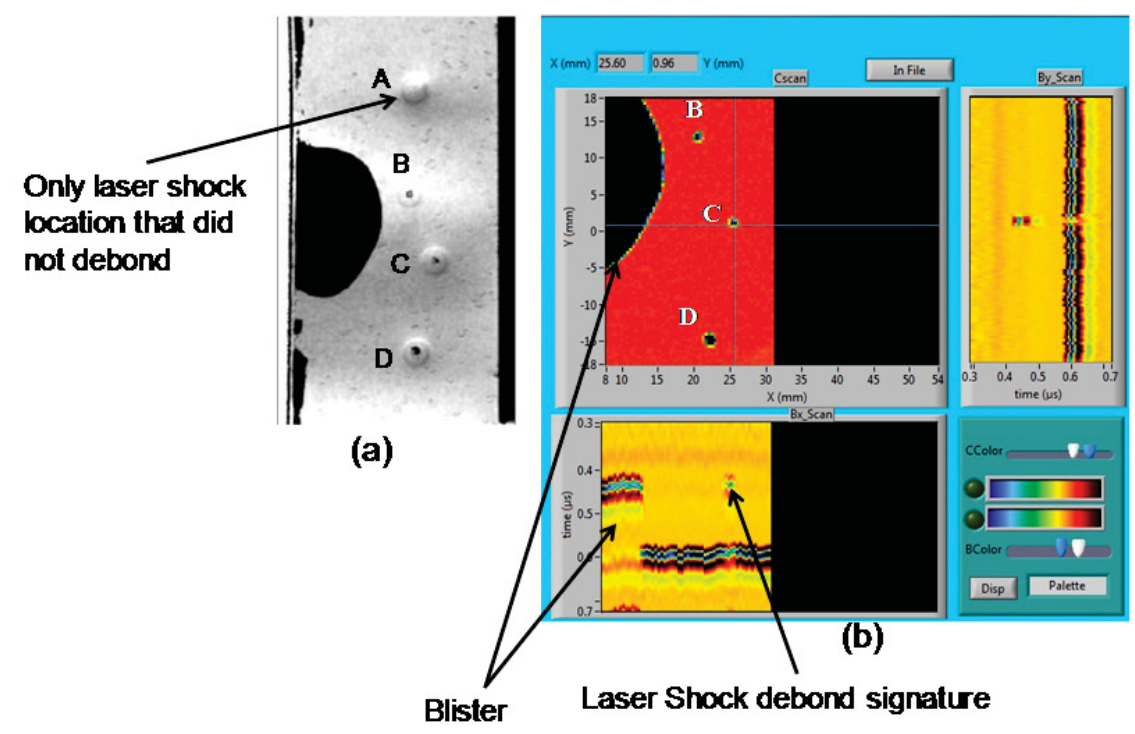

FIGURE 5. This roll bonded plate contained a weak clad-clad interface as shown by the blister in the ultrasonic C-scan image (a). Laser ultrasound images indicate the presence of debonds at the laser shock locations. The cladding interface strength appears to grow stronger away from the blister since the interface at shock location A remained intact when shocked with the same laser energy. 
The results from testing a HIP fuel plate with a DU-10Mo fuel foil is shown in Fig. 6. The images show that LST location 8 is close to the debonding threshold. Damage at location 8 can be identified in the B-scan, but the signature is not similar to a classic debond signature, as shown in Fig. 4b. It is assumed that incipient debonding has occurred in laser-shock location 8.

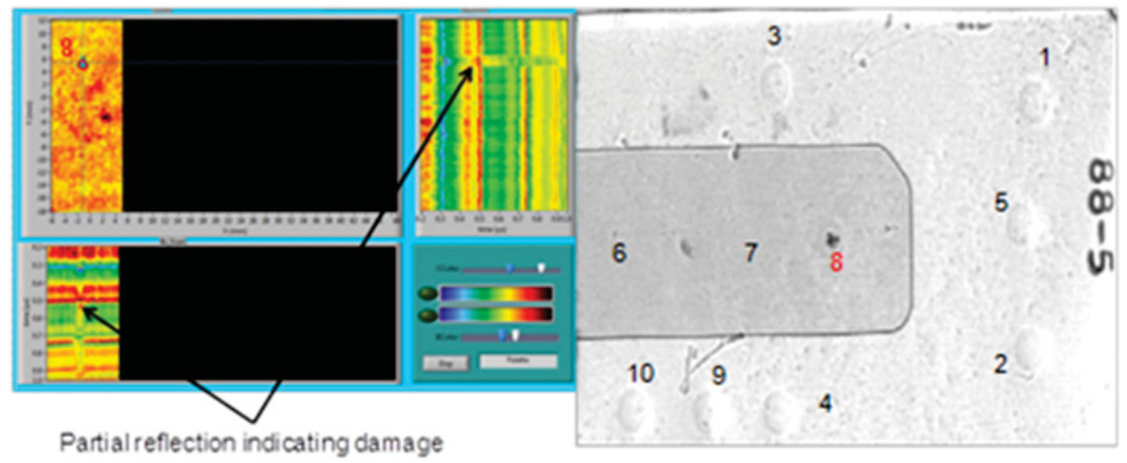

FIGURE 6. The results for fuel-clad interface in a fresh fuel plate with a DU-10Mo foil are shown. B-scan imaging shows the beginning of damage (i.e. incipient debond) at laser shock location 8 .

Friction stir welded plates have also been tested. The fuel-clad interface can be debonded just as in the HIP examples shown. However, the clad-clad interface could not be broken, similar to the the clad-clad bond on the HIPed fuel plate containing the DU-10Mo foil.

\section{DISCUSSION}

LST testing has shown the ability to debond weak clad-clad and all fuel-clad interfaces. The weak clad-clad interfaces have been produced by flawed or inadequate manufacturing processes. For all manufacturing processes, the fuel-clad interface has been successfully debonded using the LST system. This is not surprising as the bond strength of the dissimilar materials fuel-clad interfaces are expected to be lower than for the clad-clad interface. At present, the LST system has not been able to debond a well formed clad-clad interface. In such cases, it is only possible to conclude the interface strength is greater than the maximum stress value measured at the back surface. The constraining technique used in the present study is therefore adequate for fuel-clad characterization; however, a more efficient constraining mechanism will need to be developed for future clad-clad testing.

Table 1 is a summary of the interface strength characterization for clad-clad and fuel-clad interfaces in several fuel plates made by the different manufacturing processes investigated to-date. The table highlights two surprising points. Even though the constraining mechanism and maximum laser power are nominally identical for the different fuel plates, the maximum achievable stress at the back surface varies significantly. This behavior is supported by testing on homogeneous 6061 aluminum alloy samples with various tempering grades. The softest temper (6061-O) produces the higher back-surface stress than hardened alloy (6061-T6). Thus, the magnitude of the stress applied by the LST will be material-dependant.

TABLE 1. Debonding threshold measurements for clad-clad and fuel-clad interfaces in various fuel plates made by different manufacturing processes.

\begin{tabular}{|l|c|c|}
\hline \multicolumn{1}{|c|}{ Specimen } & $\begin{array}{c}\text { Clad-clad threshold } \\
\text { (MPa) }\end{array}$ & $\begin{array}{c}\text { Fuel-clad threshold } \\
\text { (MPa) }\end{array}$ \\
\hline HIP plate with SS foil using & 1,850 & 1,400 \\
Neolube parting agent & & NA \\
Roll Bonded dispersion plate & $1,500-1,600$ & 548 \\
HIP DU-10Mo fuel foil & $>1,600$ & 626 \\
HIP LEU-10Mo fuel foil & $>2,000$ & NA \\
\hline Friction stir welded SS foil & $>1,630$ & \\
\hline
\end{tabular}

The second point to be noted is that the fuel plates with "weak" interfaces - HIP with Neolube and roll-bondedactually support relatively high stresses. The stainless steel-clad interface in the HIP plate has interface strength more than 2 times the strength in the HIP plates with uranium fuel. The clad-clad interfaces in the HIP SS and roll- 
bonded dispersion plates have a debonding threshold exceeding the maximum stress obtainable using the current constraining medium.

LST does cause significant plastic deformation in the aluminum alloy, which causes localized work hardening. The plastic deformation has similar effects on the wave propagation characteristics as tempering, as shown in Table 2. The LST converts a 6061-O specimen to material that has elastic properties similar to 6061-T6

TABLE 2. Residual plastic deformation caused by the LST in 6061-0 results in the treated locations having similar ultrasonic properties to 6061-T6.

6061-O as-received :

- sample 1 (unknown)

- Thickness $1.574 \mathrm{~mm}$

- Speed $6.448971 \mathrm{~m} / \mathrm{s}$

- STD $0.002798 \mathrm{~m} / \mathrm{s}$

- $\quad$ sample 2 (spot 2)

- Thickness $1.5507 \mathrm{~mm}$

- Speed 6.442015

- STD $0.003011 \mathrm{~m} / \mathrm{s}$
6061-O after laser shock:

- 6061-O-1 (spot 1)

- Thickness $1.5653 \mathrm{~mm}$

- $\quad$ Speed $6.407069 \mathrm{~m} / \mathrm{s}$

- STD $0.003098 \mathrm{~m} / \mathrm{s}$

6061-T6 as-received

- 6061-T6 (unknown)

- Thickness $2.245 \mathrm{~mm}$

- Speed $6.38419 \mathrm{~m} / \mathrm{s}$

- STD $0.004385 \mathrm{~m} / \mathrm{s}$

\section{CONCLUSIONS}

The successful development of LEU monolithic fuel for research reactors requires a technique to characterize the strength of clad-clad and fuel-clad interfaces in both fresh and irradiated fuel plates. The laser-shock technique is currently being developed for this purpose. Currently, scoping studies are being performed to look at a variety of clad-clad and fuel-clad interfaces made from HIP, roll bonded and friction stir welding bonding processes. Test procedures and data analysis methodologies are being developed to measure bond strength. During procedure development and analysis refinement, researchers will also gain a better fundamental understanding of LST effects on various materials and interfaces. A first-order estimate of bond strength can be determined by measuring backsurface velocity just prior to reaching the debond threshold. LST testing to-date shows that interface strength can be successfully measured for the dissimilar materials fuel-clad interfaces. Clad-clad interfaces made by bonding similar materials will require the development of more efficient constraining mechanism to generate larger stresses within the material needed to cause debonding. However, LST testing has shown the ability to debond weak clad-clad interfaces.

In the future, a series of clad-clad and fuel-clad LST experiments will be conducted on fresh-fuel plates having processing histories similar to plates for which irradiation performance is known, such as archive plates from past irradiation experiments. Fuel plates from a HIP fabrication process variables study will be characterized to determine the effects of fabrication variables on as-fabricated bond strength. A study will also be performed to correlate the interface strength measured using LST with the strength measured by alternative testing techniques being developed, such as bulge testing and miniature cantilever beam testing. Finally, future work is planned to design and implement the LST technique in the hot cell environment to provide bond strength and laser-UT inspection capabilities for irradiated fuels.

\section{ACKNOWLEDGMENTS}

The funding for this work came from the US National Nuclear Security Agency which oversees the Global Threat Reduction Initiative program.

\section{REFERENCES}

1. J. L. Vossen, ASTMM Spec. Tech. Publ., 640, pp. 122-131 (1978).

2. J. Yuan, V. Gupta, A. Pronin, J. Appl. Phys. 74, pp. 2405-2410 (1993). 
3. M. Perton, A. Blouin and J.-P. Monchalin, J. Phys. D: Appl. Phys. 44 (2011).

4. Smith, James A.; Cottle, David L., Rabin, Barry H., "Laser Shockwave For Characterizing Diffusion Bonded Interfaces," to be published in AIP Conf. Proc. Vol. 151, D. O. Thompson, D. E. Chimenti, Editors, American Institute of Physics, Melville, NY, 2014.

5. Smith, James A.; Rabin, Barry H., Perton, Mathieu, Lévesque, Daniel, Monchalin, Jean-Pierre Lord, Martin, "Laser Shockwave Technique For Characterization Of Nuclear Fuel Plate Interfaces," RERTR 2012 - 34th International Meeting On Reduced Enrichment For Research And Test Reactors, Warsaw Marriott Hotel Warsaw, Poland, October 14-17, 2012.

6. M. Arrigoni, J.-P. Monchalin, A. Blouin, S. E. Kruger and M. Lord, Meas. Sci. Technol. 20, 015302 (7pp) (2009). 